\title{
Design, Realization and Challenges of an Orbital Angular Momentum Sorter: A New Instrument for Phase Microscopy
}

Amir Tavabi ${ }^{1}$, Paolo Rosi ${ }^{2}$, Giulio Pozzi ${ }^{3}$, Alberto Roncaglia ${ }^{4}$, Stefano Frabboni ${ }^{2}$, Enzo Rotunno ${ }^{5}$, Peng Han $\mathrm{Lu}^{6}$, Robert Nijland ${ }^{7}$, Peter Tiemeijer ${ }^{7}$, Moumita Ghosh ${ }^{7}$, Ebrahim Karimi ${ }^{8}$, Rafal DuninBorkowski $^{6}$ and Vincenzo Grillo ${ }^{5}$

${ }^{1}$ Ernst Ruska-Centre for Microscopy and Spectroscopy with Electrons, Jülich, Nordrhein-Westfalen, Germany, ${ }^{2}$ Università di Modena e Reggio Emilia, Modena, Emilia-Romagna, Italy, ${ }^{3}$ Università di Bologna, Bologna, Emilia-Romagna, Italy, ${ }^{4} \mathrm{CNR}-\mathrm{IMM}$, Bologna, Emilia-Romagna, Italy, ${ }^{5} \mathrm{CNR}-\mathrm{NANO}$, Modena, Emilia-Romagna, Italy, ${ }^{6}$ Forschungcentrum Juelich, Juelich, Nordrhein-Westfalen, Germany, ${ }^{7}$ ThermoFisher, Eindoven, Noord-Brabant, Netherlands, ${ }^{8}$ University of Ottawa, Ottawa, Ontario, Canada

We have realized an electrostatic "sorter" for electrons, which can be used to analyze the components of orbital angular momentum (OAM) of an electron beam [1]. It is based on the use of a combination of microscope lenses and additional customized phase elements that are positioned in the back focal plane of the objective and the first intermediate image planes (SAD aperture) of the microscope. In the stationary phase approximation, it provides a coordinate transformation between Cartesian and polar coordinates. Here, we describe the design, simulation, manufacture, computer control and alignment of the phase elements that are required to achieve OAM sorting.

The original electrostatic sorter design was introduced by McMorran [1]. The first phase element (S1) takes the form of a line of positive charge and is achieved in practice by using an electrostatic needle, which is positioned in the objective aperture plane and opens the beam to polar coordinates. The second phase element (S2) takes the form of a periodic array of alternatively biased electrodes. Figure 1 shows how the design of S1 needed to be modified to introduce astigmatism correctors [2]. Based on the results of finite element simulations, the 3D shape of the needle also had to be modified to account for inhomogeneous charging effects [3].

In order to realize the device, MEMS chips were fabricated based on silicon on insulator (SOI) technology and placed in custom aperture holders for electrical biasing. Errors in electrode shaping were corrected by using off-line focused ion beam milling. The operations of each MEMS chip was tested in the specimen position using off-axis electron holography, before inserting it into an aperture strip. Figure 2 shows a comparison of theoretical and experimental phase distributions. By using a trial and error alignment procedure, OAM spectra of test beams could be obtained with a resolution of $\mathrm{D} \ell=1.5$.

Beyond the specific application to realize an OAM sorter, we have achieved a powerful protocol for fabricating customized electrostatic phase elements and controlling them from outside the electron microscope. Applications to programmable phase plates are envisaged, with the possibility to vary an increasing number of electrodes using "fast" software controls.

This work is supported by Q-SORT, a project funded by the European Union's Horizon 2020 Research and Innovation Program under grant agreement No. 766970. 


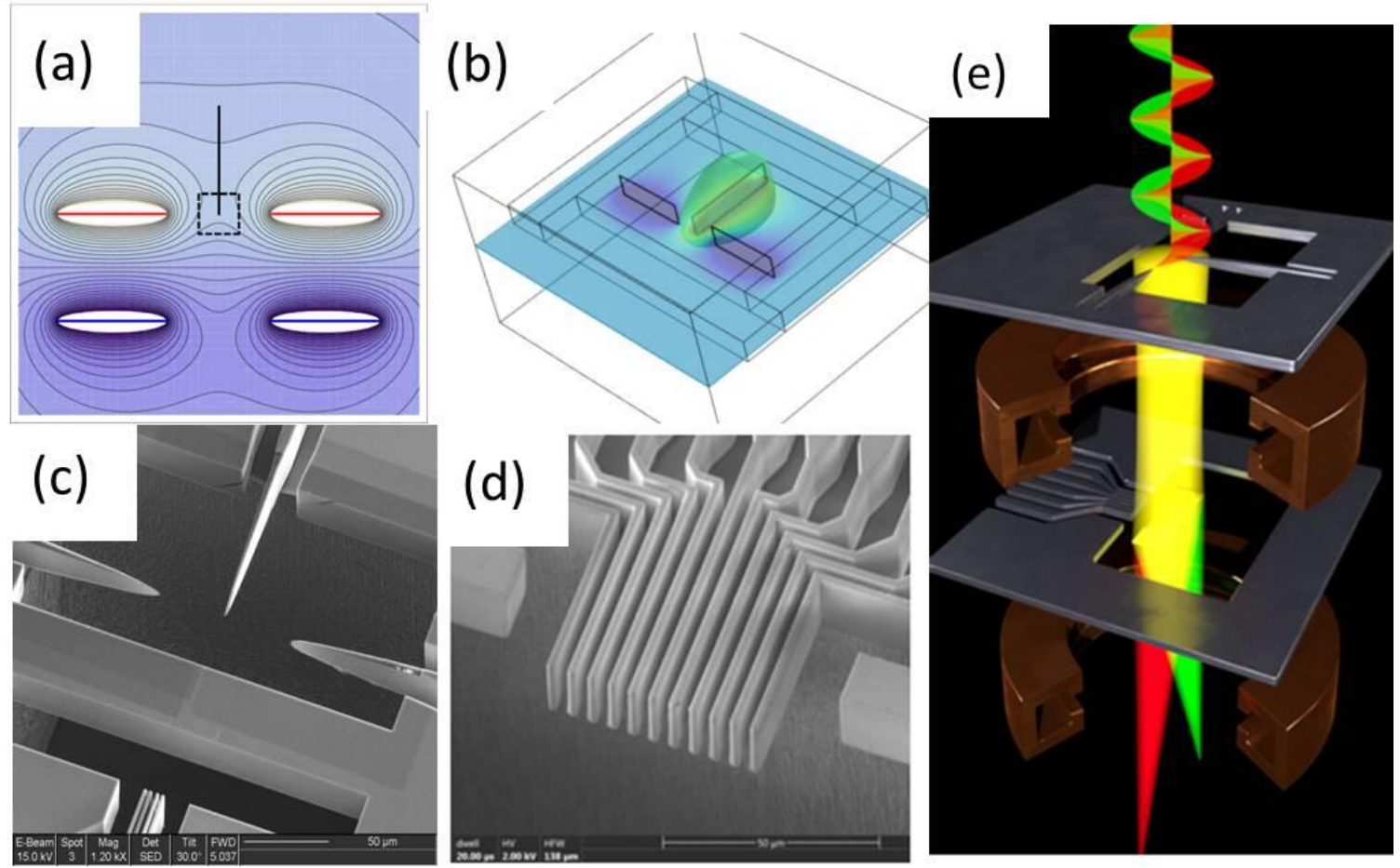

Figure 1. Selected steps in the design of sorter elements: (a) Addition of astigmatism correction using lateral needles. (b) Simulation of charge concentration. ( c) S1 realization using tapered electrodes. S2 is also shown in (d). (e) Overall scheme.

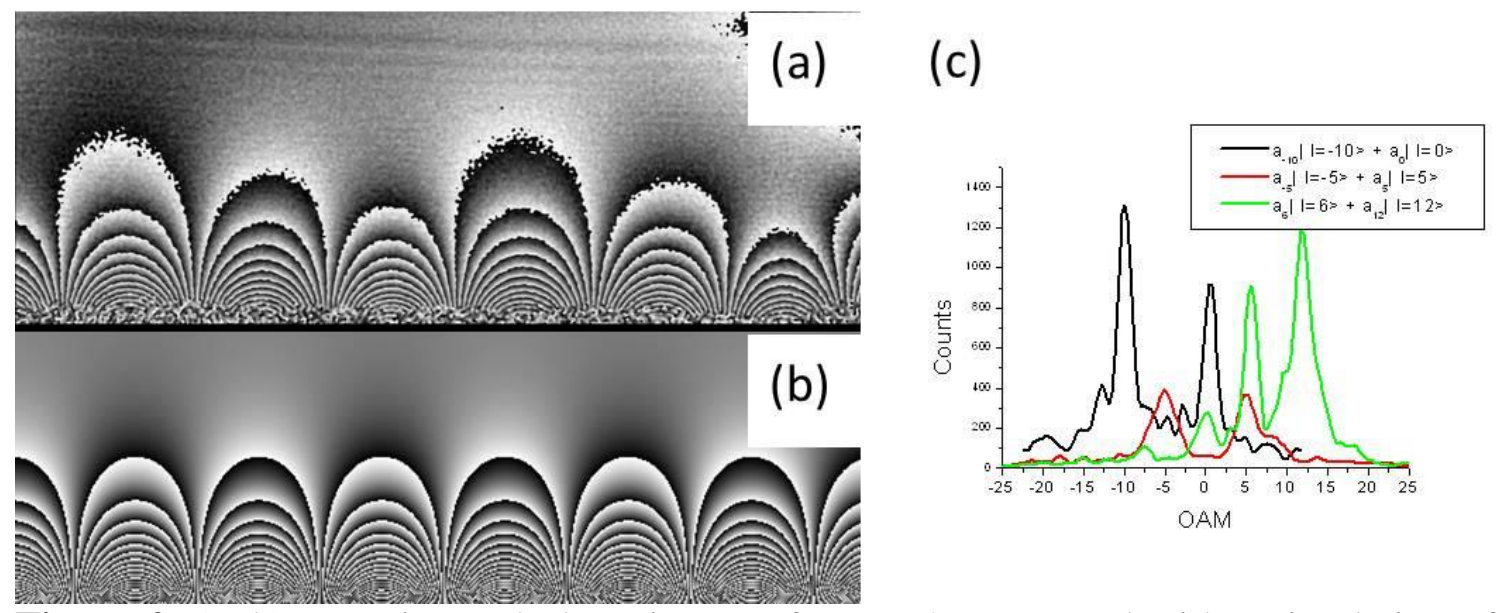

Figure 2. $(a, b)$ Experimental phase image of sorter 2, compared with a simulation of the ideal phase distribution. The period is approximately $10 \mu \mathrm{m}$. (c) Experimental OAM spectra.

\section{References}

[1] B. McMorran, T.R. Harvey, M.P.J. Lavery, New Journal of Physics 19, 023053 (2017)

[2] G. Pozzi, V. Grillo et al . Ultramicroscopy 208, 112861 (2020)

[3] A.H. Tavabi, P. Rosi et al. arXiv preprint arXiv:1910.03706 